\title{
Somatic BRCA Mutation in High Grade Epithelial Ovarian Cancer Patients
}

\author{
Tarinee Manchana ${ }^{1}$, Ruangsak Lertkhachonsuk ${ }^{1}$, Chinachote Teerapakpinyo ${ }^{2}$
}

${ }^{1}$ Division of Gynecologic Oncology, Department of Obstetrics and Gynecology, Faculty of Medicine, Chulalongkorn University and King Chulalongkorn Memorial Hospital, Bangkok, 10330, Thailand. ${ }^{2}$ Chulalongkorn GenePRO Center, Research Affairs, Faculty of Medicine, Chulalongkorn University, Bangkok, 10330, Thailand.

\begin{abstract}
Aim: To identify the frequency of somatic BRCA mutation in epithelial ovarian cancer (EOC), particularly those with high grade subtypes. Methods: Patients diagnosed with EOC included fallopian tube cancer or peritoneal cancer who had surgery during January 2015 to December 2016 were included. High grade subtypes included high grade serous carcinoma, poorly differentiated endometrioid carcinoma, and clear cell carcinoma. BRCA1 and BRCA2 mutations were tested using DNA extracted from formalin-fixed paraffin embedded block or a fresh tumor specimen then analyzed by next generation sequencing system. Patients who had no germline BRCA mutation in their peripheral blood DNA investigated by bi-directional Sanger sequencing were diagnosed as having somatic BRCA mutation. Results: 36 patients were enrolled; majority of the patients (33patients; 97.2\%) had EOC, 1 patient (2.8\%) had fallopian tube cancer and 2 patients $(5.6 \%)$ had peritoneal cancer. 28 patients (77.8\%) had high grade serous carcinoma, $6(16.7 \%)$ had poorly differentiated endometrioid carcinoma, and 2 $(5.6 \%)$ had clear cell carcinoma. BRCA1 mutation was detected in tumor tissues of 2 patients $(5.6 \%)$. These two patients had high grade serous carcinoma and significant family history of breast and/or ovarian cancers. However, BRCA1 mutations were detected in the peripheral blood in both of them. Conclusion: Only $5.6 \%$ of BRCA1 mutation was detected in ovarian tumor tissues, all mutations were found in high grade serous subtype. However, BRCA mutations were detected in the peripheral blood in both of them. Germline BRCA mutation was diagnosed, thus there were no somatic mutations in this study.
\end{abstract}

Keywords: BRCA mutation- Epithelial ovarian cancer- Somatic mutation- Thai

Asian Pac J Cancer Biol, 3 (4), 99-103

\section{Introduction}

It is estimated that at least $10 \%$ of ovarian cancers are genetically predisposition [1]. BRCA1 and BRCA2 genes are the most common susceptible genes and associated with an increased risk for both ovarian and breast cancers. Approximately $10-15 \%$ of epithelial ovarian cancer (EOC) patients have been reported to have BRCA1 or BRCA2 germline mutation [2-4]. The prevalence of BRCA mutation in high grade serous EOC is up to $20 \%$ [5]. Patients with BRCA mutated EOC have increased platinum sensitivity response rates and significant improvement in survival rate compared to those with non-BRCA mutated EOC [6]. Recently, Poly (ADP-ribose) polymerase (PARP) inhibitors have been reported to be a promising targeted therapy for BRCA deficient ovarian cancers. However,
Submission Date: 10/11/2018 Acceptance Date: 12/09/2018

it is still effective in sporadic EOC patients. Somatic mutation or epigenetic alteration in BRCA genes may be the other mechanisms. Somatic BRCA1 and BRCA2 mutations have been identified in 5-7\% of EOC patients but are more prevalent in high grade serous EOC patients. [7]. Progression free survival (PFS) in EOC patients with somatic BRCA mutation is better than patients with the wild type BRCA [8]. This result is similar to those with germline BRCA mutations. Therefore, BRCA gene testing of ovarian tumor tissue may identify more patients with EOC who may benefit from PARP inhibitors.

We have previously reported that around $11.4 \%$ of EOC patients had germline BRCA1/2 mutation [9]. However, this study focused on selected EOC patients with risk factors for hereditary ovarian cancer. These risk factors include significant family or personal history of

Corresponding Author:

Tarinee Manchana

Division of Gynecologic Oncology, Department of Obstetrics and Gynecology, Faculty of Medicine, Chulalongkorn University and King

Chulalongkorn Memorial Hospital, Bangkok, 10330, Thailand.

Email: Tarinee.M@chula.ac.th 
cancers and having a specific histological subtype such as high grade serous carcinoma. Moreover, there is no data about somatic mutation in EOC Thai patients. The objective of this study was to identify the frequency of somatic BRCA mutation in EOC patients, particularly those with high grade subtypes.

\section{Materials and Methods}

This is a cross-sectional study that was conducted between January 2015 and December 2016. Patients diagnosed with EOC included fallopian tube cancer or peritoneal cancer who had surgery during the study period were recruited. High grade subtypes included high grade serous carcinoma, poorly differentiated endometrioid carcinoma, and clear cell carcinoma. Patients with mucinous carcinoma or borderline tumor and those who had unavailable formalin-fixed paraffin embedded block (FFPE)were excluded. This study was approved by the Institutional Review Board, Faculty of Medicine, Chulalongkorn University. All patients received genetic counseling and provided written informed consents. Sample size calculation was based on previous study that reported the incidence of somatic mutation was 9\% [10]. A sample size of 32 was calculated with $95 \%$ significant level and 10\% maximum allowable error. In addition of $10 \%$ drop out, the total number was 36 patients.

BRCA1 and BRCA2 mutations were tested using DNA extracted from formalin-fixed paraffin embedded block (FFPE) or a fresh tumor specimen then analyzed by next generation sequencing system (The Illumina MiSeq System; Illumina). The enrichment of the BRCA1 and BRCA2 genes was performed using GeneRead DNAseq Human BRCA1 and BRCA2 Panel V2 according to the manufacturer's instructions (Qiagen). DNA library preparation and next generation sequencing (NGS) were performed as previously described.9 The variant pathogenicity was evaluated based on the American College of Medical Genetics and Genomics (ACMG) standard and guidelines for the interpretation of sequence variants [11]. The variants conform to the human genome variation society (HGVS) guidelines on mutation nomenclature and are referenced as sequence NM_007300.3 for BRCA1 and NM_000059.3 for BRCA2. The variant classification was listed as "Pathogenic", "Likely pathogenic", "Variant of Uncertain Clinical Significance (VUS)", "Likely benign" and "Benign" in decreasing order of clinical importance. Germline mutations in the patients who had BRCA1 or BRCA2 mutation in their tumor specimens were investigated by bi-directional Sanger sequencingusing peripheral blood DNA. Patients who had no BRCA mutation in their peripheral blood DNA were diagnosed as having somatic BRCA mutation.

\section{Results}

Thirty four FFPE and 15 fresh tumor specimens were obtained and used for DNA extraction. However, DNA could be extracted and passed quality control only in 21 FFPE (61.8\%). DNA extracted from all fresh specimens passed quality control. 36 patients were enrolled into the study. Majority of the patients had EOC (33 patients; 97.2\%). One patient $(2.8 \%)$ had fallopian tube cancer and 2 patients $(5.6 \%)$ had peritoneal cancer. Almost of all patients (97.2\%) were primary ovarian cancer; only one patient was recurrent ovarian cancer. Five patients (13.9\%) received neoadjuvant chemotherapy before primary debulking surgery. The mean age was $56.4+10.5$ years (range $37-78$ ). Twenty three patients $(63.9 \%)$ were menopausal and 14 patients $(38.9 \%)$ were nulliparous. Only 2 patients had significant history of breast cancer and/or ovarian cancer in their families. One patient had synchronous breast and ovarian cancer. Most patients presented at the advanced stage; stage $3(66.7 \%)$ and stage $4(8.3 \%)$. One-fourth of the patients had early stage cancer; $13.9 \%$ had stage 1 and $11.1 \%$ had stage 2 cancers. Twenty-eight patients $(77.8 \%)$ had high grade serous carcinoma, six (16.7\%) had poorly differentiated endometrioid carcinoma, and two $(5.6 \%)$ had clear cell carcinoma.

BRCA1 mutation was detected in tumor tissues of 2 patients $(5.6 \%)$. Details of these two patients are shown in Table 1. Both of them had high grade serous carcinoma and significant family history of cancers. One patient, aged 52 years with peritoneal cancer had a family history of breast and ovarian cancers. Her mother had breast cancer at the age of 70 years. Her elder sister had ovarian cancer at the age of 50 years. For this patient, she was found to have pathogenic BRCA1 mutation (c.3748G $>$ T, p.Glu1250Ter). Another EOC patient, aged 46 years, had pathogenic BRCA1 mutation (c.2059C > T, p.Gln687Ter). Her grandmother had ovarian cancer at the age of 70 years. For both patients, BRCA1 mutations were detected in the peripheral blood, thus there were no somatic mutations in this study but $5.6 \%$ of the patients had germline BRCA1 mutation. No BRCA mutation was identified in ovarian tissue of endometrioid and clear cell subtype. In 28 patients with high grade serous carcinoma, 2 patients $(7.1 \%)$ had germline BRCA mutation but no somatic mutation.

Variant of uncertain clinical significance (VUS) results were reported in 7 patients $(19.4 \%)$. (Table 1) Two patients had BRCA1variant (c.4370A $>$ G, p.His1457Arg), 1 had BRCA1variant (c.5135C $>$ A, p.Thr1712Lys), 1 had BRCA1 variant (c.742A $>$ G, p.Thr248Ala), 1 had BRCA1 variant (c.3344_3346delAAG, p.Glu1115del), 1 had BRCA2 variant (c. 7531 T $>$ A, p. Tyr2511Asn), and 1 patient had BRCA2 variant (c.4199A $>$ G, p.His1400Arg) and BRCA2 variant (c.6676G>A, p.Glu2226Lys).

\section{Discussion}

Testing for somatic BRCA1/2 mutation is interesting because it can increase the number of candidates that can use PARP inhibitors. PARP inhibitors have been reported to improve PFS in recurrent platinum sensitive ovarian cancer patients [12]. Thus, PARP inhibitors were licensed by European Medicines Agency (EMA) for both germline and somatic BRCA mutation in ovarian cancer patients. Around $18 \%$ of ovarian tumor tissues harbor 


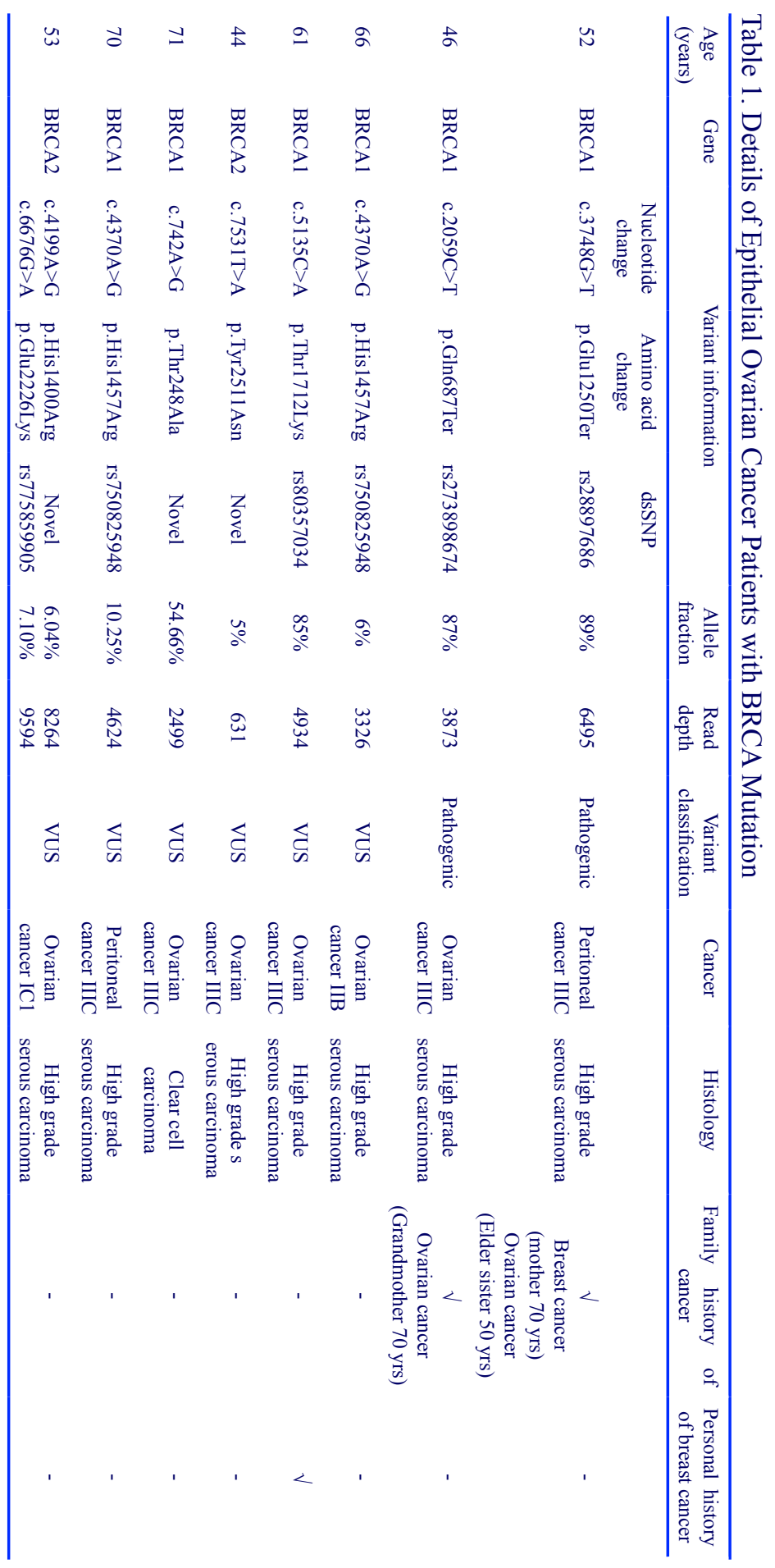

BRCA1/2 mutation but around $23 \%$ of high grade serous tumors [8]. After analyzing the germline DNA, somatic BRCA1/2 mutation was identified in $5-7 \%$ of EOC [7-8]. No BRCA1/2 mutation was reported in grade 1 tumors or non-serous subtype of ovarian cancer tissue [8-13]. This result is similar to our study; EOC patients with poorly differentiated endometrioid or clear cell carcinoma did not have BRCA1/2 mutations. The prevalence of BRCA mutation might vary by ethnicities and countries. There are few studies about germine BRCA mutation in Asian countries, which reported the frequency of BRCA mutation between 5-26\% [13-18]. Korean had the highest frequency. However, these studies were not a large cohort. They might have selection bias and different techniques of gene mutation analysis. There are limited studies of somatic mutation in EOC in Asian population. No somatic mutation was reported in Japanese EOC patients but about 4\% in Taiwanese patients [13-16]. All mutations were detected in serous subtype [16]. These finding is inconsistent with our study; somatic mutation was unable to identify in all ovarian cancer tissue despite of serous subtype. It is possible that the sample size may have been too small to allow for such detection. Furthermore, tumor tissues from primary and recurrent setting might be different frequency of mutation. Almost of all tumor tissues in our study were primary 
cancers, which might be plausible explanation for negative finding in this study.

High grade serous carcinoma is the most common subtype worldwide; its incidence is around 70\% [5]. The prevalence for high grade serous carcinoma is much lower in Thailand; its incidence is $20-25 \%$ in Thai ovarian cancer patients [9-19]. On the other hand, the proportion of endometrioid and clear cell carcinoma are more frequent, up to $65 \%$, whereas mucinous carcinoma was reported about $20 \%$ of them [19]. This may be another plausible explanation for the differences. Our previous study reported the frequency of germline BRCA mutation about $11.4 \%$ in selected EOC patients with history of breast and/ or ovarian cancer or personal history of breast cancer [9]. Thus, the frequency of BRCA mutation might be lower in unselected EOC patients in Thailand. This assumption was confirmed in the current study that only $5.6 \%$ of patients had germline BRCA mutation and no patients had somatic mutation.

There are 2 strategies for genetic testing. Most countries continue to use germline testing as the first line testing; tumor specimens will be further tested if the test results are negative. Alternatively, genetic testing of the tumor specimen is used as a screening tool. If the test is positive, additional tests are done to confirm if the mutations are germline or somatic. In contrast, if the gene mutations are not found in the tissues, further testing for germline mutation is not required. This strategy may be more cost effective and can reduce the workload of the geneticists. One drawback for this approach is the process of tissue fixation in FFPE. The fixation of the tissue should be optimized otherwise it will affect the quality of the extracted DNA. Our study showed only $62 \%$ of FFPE specimens within one year after surgery could provide good quality DNA which can be used for further analysis. This number might be decreased in FFPE which were stored for longer period. Nowadays, PARP inhibitors have been approved for maintenance treatment in recurrent platinum sensitive ovarian cancer. It is unlikely to have recurrent tumor tissue for somatic mutation testing. The status of BRCA mutation in tumor tissue from primary setting may not be concordance to the mutation in recurrent setting.

Only $5.6 \%$ of BRCA1 mutation was detected in ovarian tumor tissues. All mutations were found in high grade serous subtype, and none in endometrioid or clear cell carcinoma. BRCA mutations were detected in the peripheral blood of these two patients. Therefore, germline BRCA1 mutation was diagnosed and there was no somatic mutation in this study. However, the negative result in this study might be from small sample size. Further study with larger number of samples is necessary to confirm different BRCA mutation in different ethnicities. Although, there is limited number of geneticists, genetic testing in tumor specimen is still not widespread use in Thailand. Fresh tumor specimen is not routinely collected and uncertain quality of the extracted DNA from fixation process may be possible barrier. Furthermore, high grade serous carcinoma is not common subtype in Thai, testing of BRCA mutations in ovarian tumor tissues may have limited role in Thailand.

Disclosure

All authors declared no conflicts of interest.

\section{References}

1. Russo A, Calò V, Bruno L, Rizzo S, Bazan V, Di FedeG. Hereditary ovarian cancer. Crit Rev Oncol Hematol 2009;69:28-44.

2. Zhang S, Royer R, Li S, McLaughlin JR, Rosen B, Risch HA, et al. Frequencies of BRCA1 and BRCA2 mutations among 1,342 unselected patients with invasive ovarian cancer. Gynecol Oncol 2011;121:353-7.

3. Alsop K, Fereday S, Meldrum C, deFazio A, Emmanuel C, George J, et al. BRCA mutation frequency and patterns of treatment response in BRCA mutation-positive women with ovarian cancer: a report from the Australian Ovarian Cancer Study Group. J Clin Oncol 2012;30:2654-63.

4. Song H, Cicek MS, Dicks E, Harrington P, Ramus SJ, Cunningham JM, et al. The contribution of deleterious germline mutations in BRCA1, BRCA2 and the mismatch repair genes to ovarian cancer in the population. Hum Mol Genet 2014;23:4703-9.

5. Ledermann JA, Drew Y, Kristeleit RS. Homologous recombination deficiency and ovarian cancer. Eur J Cancer 2016;60:49-58.

6. Pennington KP, Walsh T, Harrell MI, Lee MK, Pennil CC, Rendi $\mathrm{MH}$, et al. Germline and somatic mutations in homologous recombination genes predict platinum response and survival in ovarian, fallopian tube, and peritoneal carcinomas. Clin Cancer Res 2014;20:764-75.

7. Moschetta M, George A, Kaye SB, Banerjee S. BRCA somatic mutations and epigenetic BRCA modifications in serous ovarian cancer. Ann Oncol. 2016;27:1449-55.

8. Hennessy BT, Timms KM, Carey MS, Gutin A, Meyer LA, Flake DD2nd, et al. Somatic mutations in BRCA1 and BRCA2 could expand the number of patients that benefit from poly (ADP ribose) polymerase inhibitors in ovarian cancer. J Clin Oncol 2010;28:3570-76.

9. Chirasophon S, Manchana T, Teerapakpinyo C. High-risk epithelial ovarian cancer patients for hereditary ovarian cancer. J Obstet Gynaecol Res 2017;43:929-34.

10. Dann RB, DeLoia JA, Timms KM, Zorn KK, Potter J, Flake DD 2nd, et al. BRCA1/2 mutations and expression: response to platinum chemotherapy in patients with advanced stage epithelial ovarian cancer. Gynecol Oncol 2012;125:677-82.

11. Richards S, Aziz N, Bale S, Bick D, Das S, Gastier-Foster $\mathrm{J}$, et al. Standards and guidelines for the interpretation of sequence variants: a joint consensus recommendation of the American College of Medical Genetics and Genomics and the Association for Molecular Pathology. Genet Med 2015;17:405-24.

12. Wiggans AJ, Cass GK, Bryant A, Lawrie TA, Morrison J. Poly(ADP-ribose) polymerase (PARP) inhibitors for the treatment of ovarian cancer. Cochrane Database Syst Rev. 2015 May 20;(5):CD007929.

13. Matsushima M, Kobayashi K, Emi M, Saito H, Saito J, Suzumori K, et al. Mutation analysis of the BRCA1 gene in 76 Japanese ovarian cancer patients: four germline mutations, but no evidence of somatic mutation. Hum Mol Genet 1995;4:1953-6.

14. Khoo US, Ngan HY, Cheung AN, Chan KY, Lu J, Chan $\mathrm{VW}$, et al. Mutational analysis of BRCA1 and BRCA2 genes in Chinese ovarian cancer identifies 6 novel germline 
mutations. Hum Mutat 2000;16:88-9.

15. Choi MC, Heo JH, Jang JH, et al. Germline Mutations of BRCA1 and BRCA2 in Korean Ovarian Cancer Patients: Finding Founder Mutations. Int J Gynecol Cancer 2015;25:1386-91.

16. Chao A, Chang TC, Lapke N, Jung SG, Park H, JooWD, et al. Prevalence and clinical significance of BRCA1/2 germline and somatic mutations in Taiwanese patients with ovarian cancer. Oncotarget 2016;7:85529-41.

17. Hasmad HN, Lai KN, Wen WX, Park DJ, Nguyen-Dumont T, Kang PC, et al. Evaluation of germline BRCA1 and BRCA2 mutations in a multi-ethnic Asian cohort of ovarian cancer patients. Gynecol Oncol 2016;141:318-22.

18. Sakamoto I, Hirotsu Y, Nakagomi H, Ouchi H, Ikegami A, TeramotoK, et al. BRCA1 and BRCA2 mutations in Japanese patients with ovarian, fallopian tube, and primary peritoneal cancer. Cancer 2016;122:84-90.

19. Manchana T, Kobwitaya K. Survival outcomes for different subtypes of epithelial ovarian cancer. Clin Res Obstet Gynecol 2018;1:1-7.

\section{(ब) $(9$}

This work is licensed under a Creative Commons AttributionNon Commercial 4.0 International License. 